3

4

5

6

\section{Multi-enveloping of particulated antigens with biopolymers and} immunostimulant polynucleotides

J.F. Correia-Pinto ${ }^{\mathrm{a}, \mathrm{b}}$, Mercedes Peleteiro ${ }^{\mathrm{c}}$, N. Csaba ${ }^{\mathrm{a}, \mathrm{b}}$, África González-Fernández ${ }^{\mathrm{c}}$, M.J. Alonso ${ }^{\mathrm{a}, \mathrm{b}}$

a Department of Pharmacy and Pharmaceutical Technology, School of Pharmacy, University of Santiago de Compostela, 15782 Santiago de Compostela, Spain

${ }^{\mathrm{b}}$ Center for Research in Molecular Medicine and Chronic Diseases (CIMUS), Av. Barcelona s/n, Campus Vida, University of Santiago de Compostela, 15707 Santiago de Compostela, Spain

'Immunology, Institute of Biomedical Research (IBIV), Biomedical Research Center (CINBIO), University of Vigo, Vigo, Spain

*Corresponding author at: Department of Pharmacy and Pharmaceutical Technology, School of Pharmacy, University of Santiago de Compostela, 15782 Santiago de Compostela, Spain. Tel.: +34 881815252; fax: +34 981547148.

E-mail address: mariaj.alonso@usc.es (M.J. Alonso).

\section{Keywords}

Hepatitis B; VLP; Multi-enveloped nanoparticle; Polyamino acid; Polysaccharide; Poly (I:C) 
23 Abstract

24 The main objective of this work is to study the possibility of enveloping particulated

25 antigens using biomaterials and polynucleotides with immunostimulant properties, and

26 to assess the value of this potential adjuvant strategy. The nanoparticulate structure (22

$27 \mathrm{~nm}$ ) of the recombinant Hepatitis B surface antigen (rHBsAg) has inspired us to use it

28 as a substrate in the design of multi-enveloped nanoparticles. Our approach relies on

29 the adsorption of polymers and immunostimulants by electrostatic interactions.

30 Considering the negative charge of the rHBsAg, as a first layer we adsorbed the

31 cationic polymers protamine (PR) and polyarginine (PARG). Subsequently, these

32 nanostructures were enveloped using the anionic polymers dextran sulfate (DS) and

33 alginate (ALG), and the immunostimulant polynucleotide poly (I:C) (pIC). The

34 resulting multi-enveloped nanoparticles (HB:PR; HB:PR:DS; HB:PARG:ALG;

35 HB:PARG:pIC) presented very small sizes $(<50 \mathrm{~nm})$ and were shown to be

36 internalized by macrophages. With regard to the capacity of these new

37 nanocompositions to elicit immune responses, the results observed following either

38 intranasal or intramuscular administration indicated that despite the modest IgG titers,

39 the IgG1/IgG2a ratio was more balanced that the one observed for the alum-adsorbed

40 antigen. Overall, this work highlights the possibility to protect antigens and modify

41 their presentation using a simple layer-by-layer approach. 
44 Vaccination is the most cost-effective preventive intervention against disease to date

45 [1], and while the majority of the marketed vaccines are based on attenuated and inactivated whole-cell vaccines, the current tendency is to design vaccines based on subunit antigens. These subunit vaccines are based on specific components of pathogens, and are less prone to provoke adverse reactions [2]. Among the subunit antigens, virus-like particles (VLP), formed by self-assembled viral proteins, are particularly interesting due to their superior immunogenicity [3, 4]. Notable examples of VLP-based vaccines are the current hepatitis B (HB) and the human papillomavirus (HPV) vaccines, which are based on the recombinant hepatitis B surface antigen (rHBsAg) [5] and on the HPV L1 protein, respectively [6]. In addition, other VLPbased vaccines, such as RTS,S for malaria and ACAM-FLU-A for Influenza A, are currently in the clinical development stage [7]. Despite their immunogenicity, these particulated antigens still need to be associated to a suitable vaccine adjuvant in order to generate an effective immune response. Most commonly, subunit antigens are adsorbed onto alum salts as adjuvant, due to their long record of safe use and effectiveness [8]. However, these adjuvants present several limitations. The use of alum requires a tight control of the storage conditions, as freezing provokes aggregation and loss of effectiveness [9]. This is a particularly important problem because the maintenance of the global cold chain has significant limitations [10] and can lead to a decreased immunization coverage worldwide [11]. Additionally, the use of alum is limited to parenteral administration, which requires specific training to perform and can cause blood-borne pathogen contamination by the re-use of syringes [12]. Finally, alum salts generate $\mathrm{a}_{\mathrm{h}} 2$ biased immune response and they lack the capacity to stimulate cell-mediated immunity [13].

The use of nanotechnology-based approaches is known to offer opportunities for improving the formulation of vaccines. Specific advantages of the nanoformulations described in the literature include: i) their improved thermostability, as compared to the alum-adjuvanted vaccines $[14,15]$; ii) their capacity to facilitate the transport of antigens across mucosal barriers and generate humoral and mucosal responses [15, 16]; iii) their ability to enhance and prolong the immune responses (single-dose vaccination) [17]; iv) their potential to modulate $\mathrm{T}_{\mathrm{h}} 1 / \mathrm{T}_{\mathrm{h}} 2$ immune responses [16, 17]. 
It has been hypothesized that nanocarriers may facilitate antigen presentation to $\mathrm{T}$ cells due to their resemblance to virus in terms of size and surface characteristics $[18,19]$. These antigen-loaded nanocarriers are captured in the administration area by local antigen-presenting cells (APC), particularly dendritic cells (DCs) [20]. Then, DCs process the antigen while migrating to the lymph nodes through the lymphatic vessels [18]. In the lymph nodes, once the antigen is processed, the DCs present individual peptides associated to the major histocompatibility complex (MHC) class II molecules to specific T lymphocytes, triggering the adaptive immune response, with the generation of memory $\mathrm{T}$ and $\mathrm{B}$ cells [21]. In addition to this peripheral uptake of antigen-loaded nanocarriers by DCs, it has been recently proposed that small nanoparticles $(<100 \mathrm{~nm}$ ) could target antigens directly to the DCs in the lymph nodes $[18,22,23]$. As the immune cell population in these locations is much more concentrated that in peripheral tissues [24], such strategy could potentially provide a better and higher antigen presentation, increasing the immune response and decreasing local reactions to the vaccine $[23,25]$. An additional advantage of these ultra-small nanocarriers, as compared to larger particles or alum- based vaccines, relies on the fact that they could be sterilized by filtration.

Despite these data, most of the antigen delivery carriers described until now present a size larger than $100 \mathrm{~nm}$ [26]. Therefore, acknowledging this technological gap, our main goal was to rationally design enveloped virus-like particle antigens with a final size of less than $50 \mathrm{~nm}$. For this purpose, our approach has been to use the antigen itself as a substrate for the adsorption of polymers and polynucleotides with adjuvant properties. As a model antigen we have chosen the recombinant hepatitis B surface antigen (rHBsAg) in its VLP form [5]. This antigen is formed by the self-assembly of rHBsAg proteins with associated lipids, and presents an average particle diameter of $22 \mathrm{~nm}$ with negative surface charge [27]. Because of this negative surface charge, we have selected for the first enveloping layer two different cationic polymers: polyarginine (PARG) and protamine (PR). The second enveloping layer consisted of anionic polysaccharides, such as dextran sulfate (DS) or alginate (ALG), or a synthetic double-stranded based RNA, poly (I:C) (pIC).

The rational for the selection of the polymers was as follows. Polyarginine is a cationic polyamino acid, which has been shown to favor the uptake of antigens by 
antigen-presenting cells [28] and, as a consequence, to enhance the T cell responses in vivo against associated antigens [29]. These interesting adjuvant properties have led to its inclusion in a hepatitis $\mathrm{C}$ vaccine (HCV), which is currently being tested in clinical trials [30]. On the other hand, protamine is a mixture of arginine-rich polypeptides, with membrane translocating properties [31], which has also shown the capacity to increase the uptake of both microparticles [32] and nanoparticles by phagocytic cells [31]. With regard to the selection of the negatively charged polysaccharides, dextran sulfate and alginate are both linear anionic polymers exhibiting immunoadjuvant properties. Alginate has been found to activate macrophages [33] inducing the production of pro-inflammatory cytokines [34], possibly through the interaction with Toll-like receptors (TLR) [35]. There is also evidence of the capacity of dextran sulfate to increase cell-mediated immunity [36] and antibody responses [37] in several animal models. Finally, the polynucleotide poly (I:C) is an agonist of the Toll-like receptor 3 (TLR-3) present in dendritic cells. TLR-3 recognizes double-stranded RNA (dsRNA) associated to viral infection, stimulates dendritic cell activation and maturation and the secretion of interferon alpha/beta, which, in turn, plays an important role in the defense against viruses [38]. Through these mechanisms, poly (I:C) can potentiate both humoral and cellular immune responses [39, 40]. The adsorption of the poly (I:C) onto the enveloped antigen was thought to be particularly important in order to achieve simultaneous delivery of the antigen and the immunostimulant to the immune cells.

In brief, this work describes the development of small nanovaccines $(<50 \mathrm{~nm})$ through a multi-enveloping method using HB VLP as model antigen, and their characterization through the study of their physicochemical properties, uptake by immune cells and in vivo capacity to generate an effective immune response to the $\mathrm{HB}$ antigen after intramuscular (i.m.) and intranasal (i.n.) administration.

\section{Material and methods}

\subsection{Materials}

Poly-L-arginine hydrochloride with molecular weight of 5 - 15 kDa (P4663), dextran sulfate sodium salt with molecular weight of 9 - $20 \mathrm{kDa}$ (D6924) and Phosphate Buffer saline (PBS) were obtained from Sigma-Aldrich (Madrid, Spain). Alginate with molecular weight of $4 \mathrm{kDa}$ was obtained from Danisco (Copenhagen, Denmark). 
145 Protamine sulfate was kindly donated by LEO Pharma (Denmark). Polyinosinic-

146 polycytidylic acid of low molecular weight (0.2 - $1 \mathrm{kB}$ ) (Poly (I:C)-LMW) was

147 purchased from Invivogen (San Diego, USA). The recombinant hepatitis B surface

148 antigen (rHBsAg) was kindly donated by Shantha Biotechnics Ltd. (Hyderabad, India)

149 as an aqueous suspension in PBS with a protein concentration of $1.13 \mathrm{mg} / \mathrm{ml}$.

150 The 5-carboxytetramethylrhodamine, succinimidyl ester (5-TAMRA, SE) *single

151 isomer* (Catalogue \# C2211) and the Slide-A-Lyzer Dialysis Cassettes, 2K MWCO

152 were purchased from ThermoFisher Scientific (Massachusetts, USA). Amicon Ultra-4

$153 \mathrm{ml}$ Centrifugal Filters with $100 \mathrm{kDa}$ pore and low-binding regenerated cellulose

154 membrane were purchased to Merck Millipore (Massachusetts, USA).

155

156

157

158

159

160

161

162

163

164

165

\subsection{Preparation of multi-enveloped particles using hepatitis $B$ antigen as} substrate

An aliquot of the antigen suspension diluted with ultrapure water was introduced in an Amicon-4 $100 \mathrm{kDa}$ filtration tube and centrifuged at 5,000 rpm for 5 minutes at $15^{\circ} \mathrm{C}$ in a Universal 32 centrifuge (Hettich, Tuttlingen, Germany). After repeating this procedure three times, the antigen was recovered from the filter and diluted to the final concentration of $0.1 \mathrm{mg} / \mathrm{ml}$. This final solution was then used as template for the adsorption of the first polymer layer.

\subsubsection{Preparation of HB:PR and HB:PARG - First layer adsorption}

A volume of $0.8 \mathrm{ml}$ of protamine or polyarginine solution $(0.1 \mathrm{mg} / \mathrm{ml})$ was mixed in a glass tube with $0.8 \mathrm{ml}$ of HB solution $(0.1 \mathrm{mg} / \mathrm{ml})$ under magnetic stirring (200 rpm). The magnetic stirring was maintained for 5 minutes. The resulting suspension was incubated for 20 minutes at room temperature in order to allow the formation of the complex. The formulation was filtered then, using an Amicon-4 100 kDa and diluted to a final volume of $4 \mathrm{ml}$ with ultrapure water. This ultrafiltration procedure was repeated 3 times and subsequently the concentrated dispersion of the HB:PR or HB:PARG complexes was recovered and diluted to a final HB concentration of 0.1 $\mathrm{mg} / \mathrm{ml}$. 


\subsubsection{Preparation of HB:PR:DS, HB:PARG:ALG and HB:PARG:pIC - Second} layer adsorption

A volume of $0.785 \mathrm{ml}$ of dextran sulfate $(0.4 \mathrm{mg} / \mathrm{ml})$, alginate $(0.4 \mathrm{mg} / \mathrm{ml})$ or poly (I:C) $(0.1 \mathrm{mg} / \mathrm{ml})$ solution was mixed in a glass tube with $0.785 \mathrm{ml}$ of HB:PR or HB:PARG formulation (HB at $0.1 \mathrm{mg} / \mathrm{ml}$ ) under magnetic stirring (200 rpm) to constitute the HB:PR:DS, HB:PARG:ALG or the HB:PARG:pIC formulation, respectively. The formulations were kept under stirring for 5 minutes and then they were incubated at room temperature for 20 minutes. As in the case of the first layer, the formulations from the previous step were filtered with an Amicon-4 $100 \mathrm{kDa}$ and diluted to a final volume of $4 \mathrm{ml}$. This ultrafiltration procedure was repeated three times and subsequently the concentrated dispersion of the complexes HB:PR:DS, HB:PARG:ALG and HB:PARG:pIC was recovered and diluted to a final HB concentration of $0.1 \mathrm{mg} / \mathrm{ml}$. The final theoretical mass ratio for HB:PR:DS, HB:PARG:ALG and HB:PARG:pIC was estimated as 1:1:4, 1:1:4 and 1:1:1, respectively.

\subsection{Physicochemical characterization of the multi-enveloped particles}

The particle size and polydispersity index (PdI) of the multi-enveloped particles were evaluated by Dynamic Light Scattering (DLS) and its surface charge (zeta potential) by Electrophoretic Light Scattering (ELS), using a Zetasizer Nano ZS90 (Malvern Instruments, UK). The measurements were performed at $25^{\circ} \mathrm{C}$ with a detection angle of $173^{\circ}$, in ultrapure water.

\subsection{Determination of protamine and polyarginine adsorption and antigen enveloping efficiency}

The protamine and polyarginine adsorption to the HB particles was evaluated by an indirect measurement based on the free polyamino acid in solution after incubation with the antigen (micro BCA protein assay, Pierce, Thermo Fisher Scientific, MA, USA). The efficiency of adsorption (E.A.\%) was calculated subtracting the concentration determined by the micro BCA from the theoretical concentration of the polyamino acid $(0.1 \mathrm{mg} / \mathrm{ml})$.

The amount of antigen coated by the polymers was determined indirectly using a microtiter plate-based sandwich ELISA kit (Murex HBsAg Version 3, Murex Biotech Ltd.; Dartford, UK). The HB enveloping efficiency (E.E.\%) was calculated by the 
212 difference between the theoretical amount of antigen and the amount of non-enveloped

213 antigen determined in suspending medium. It was assumed that the enveloped antigen

214 particles with polymers, when incubated in the microwells, would not be recognized

215 by the anti-HB antibodies of the ELISA assay.

216

217

218

\subsection{Stability of the multi-enveloped particles in aqueous suspension}

\subsubsection{Stability during storage}

219

The multi-enveloped nanoparticles, HB:PR 1:1, HB:PR:DS 1:1:4, HB:PARG:ALG 1:1:4 and HB:PARG:pIC 1:1:1, at HB concentration of $0.1 \mathrm{mg} / \mathrm{ml}$, were stored at $4{ }^{\circ} \mathrm{C}$ for up to 1 month. Then their size and PdI were determined, as described in section 2.3.

\subsubsection{Stability upon dispersion in simulated biological medium}

The stability of the multi-enveloped nanoparticles HB:PR 1:1, HB:PR:DS 1:1:4. HB:PARG:ALG 1:1:4 and HB:PARG:PIC 1:1:1, at HB concentration of $0.05 \mathrm{mg} / \mathrm{ml}$, in PBS at $37^{\circ} \mathrm{C}$, was tested for up to 4 hours.

\subsection{Preparation of fluorescently labeled HB:PR:DS and HB:PARG:ALG multi-} enveloped particles

In order to perform in vitro cell uptake studies, HB:PR:DS and HB:PARG:ALG particles were prepared with fluorescently labeled protamine (PRF) and polyarginine (PARGF), respectively. The dye chosen was the 5-carboxy tetramethylrhodamine succinimidyl ester (TAMRA) and the labeling was performed according to the manufacturer's instructions. Briefly, protamine and polyarginine were dissolved in 0.1 M sodium bicarbonate buffer, $\mathrm{pH}$ 8.0, at $10 \mathrm{mg} / \mathrm{ml}$. In parallel, TAMRA was dissolved in dimethyl sulfoxide (DMSO) at $10 \mathrm{mg} / \mathrm{ml}$. An aliquot of the TAMRA solution (0.1 $\mathrm{ml}$ ) was added slowly over $1 \mathrm{ml}$ of the polyamino acid solution under magnetic stirring and left for 1 hour at room temperature. After incubation, the labeled polyamino acid solution was dialyzed in Slide-A-Lyzer Dialysis Cassette 2K MWCO for $48 \mathrm{~h}$, to remove free TAMRA. After dialysis, the labeled protamine (PRF) or polyarginine (PARGF) was adsorbed onto the HB antigen and the polysaccharide dextran sulfate or alginate was added over the PRF or PARGF layers to form the

244 fluorescently labeled HB:PRF:DS or HB:PARGF:ALG particles, respectively. The 
245 labeled formulations were then characterized in terms of size, PdI and surface charge, 246 as described in 2.3.

$248 \quad$ 2.7. In vitro cell studies

$249 \quad$ 2.7.1. Cells and culture

250 The adherent RAW 264.7 cells, a murine macrophage cell line (ATCC, Manassas,

251 VA), were cultured in RPMI supplemented with 10\% (v/v) fetal bovine serum (FBS)

252 (PAA; Pasching, Austria), $2 \mathrm{mM}$ glutamine and $100 \mathrm{U} / \mathrm{ml}$ of penicillin/streptomycin, 253 in a humidified atmosphere at $37^{\circ} \mathrm{C}$ in 5\% carbon dioxide atmosphere. Cells were 254 split every other day to maintain $70-80 \%$ of confluent cultures.

\subsubsection{Internalization studies in macrophages}

The uptake of HB:PR:DS and HB:PARG:ALG by Raw 264.7 cells was examined using a confocal microscope (Leica SP5, Germany). Briefly, cells (3 x 105) were seeded on a glass cover-slip (Menzel-Gläser; Braunschweig, Germany) in a 24-well plate (Falcon 3047, BD Biosciences, USA) and left overnight at $37^{\circ} \mathrm{C}$ to allow cell adhesion. The following day, cells were incubated with TAMRA-labeled HB:PRF:DS or TAMRA-labeled HB:PARGF:ALG formulation (in a mass equivalent to $10 \mu \mathrm{g}$ of $\mathrm{HB}$ ), in RPMI with $10 \% \mathrm{FBS}$ for 30 min at $37^{\circ} \mathrm{C}$, in the dark. After several washes with PBS to remove non-internalized particles, the cells were fixed with $4 \%$ formaldehyde for $10 \mathrm{~min}$ and then incubated with Alexa Fluor 488-phalloidin (Invitrogen, Thermo Fisher Scientific, USA) for 20 min to stain the cellular cytoskeleton. Finally, the cover-slips containing the attached cells were mounted on slides in the presence of ProLong ${ }^{\circledR}$ Gold Antifade mounting medium (Invitrogen, Thermo Fisher Scientific, USA), containing DAPI as nucleus stain, and analyzed in a confocal microscope (Confocal Laser Scanning Microscopy (Leica SP5)).

\subsection{In vivo studies}

\subsubsection{Animals}

274 Female BALB/c mice (4-5 weeks) were housed in filter-top cages in a $12 \mathrm{~h} \mathrm{light/12} \mathrm{h}$

275 dark cycle at constant temperature $\left(22^{\circ} \mathrm{C}\right)$ and provided with a standard diet and water

276 ad libitum. The animals were kept conscious during the immunizations and bleeding.

277 All protocols were in accordance with the guidelines of the Spanish regulations (Royal 
278 Decree 1201/2005) regarding the use of animals in scientific research and under the

279 approval of the Ethical Committee of the University of Vigo.

2.8.2. Preparation of the control vaccine using alum as adjuvant.

The HB antigen was used either suspended in PBS for intranasal (i.n.) administration or adsorbed on aluminum hydroxide (HB-Alum) for intramuscular (i.m.) administration, as positive control. Briefly, Alum (Alhydrogel ${ }^{\circledR}$, Sigma-Aldrich, St. Louis, MO) was incubated with the HB antigen in a 3:1 (v:v) ratio for 30 minutes at 4 ${ }^{\circ} \mathrm{C}$ under gentle agitation. Afterwards, the suspension was centrifuged (10,000 x g, 10 minutes, $4^{\circ} \mathrm{C}$ ) and the pellet was resuspended in an isotonic saline solution. The dose of antigen administered $(10 \mu \mathrm{g})$ was the same in the control vaccines and in the rest of formulations.

290

\subsubsection{Immunization and bleeding schedule}

In total, three in vivo trials were performed in mice, in order to evaluate the capacity of 1) the HB:PR, 2) the HB:PR:DS and HB:PARG:ALG and 3) the HB:PARG:pIC systems, to stimulate an immune response against the HB antigen by i.n. and i.m. routes.

296 In all cases, the initial pool of mice was randomly distributed in groups of ten animals for each formulation and administration route (i.m. or i.n.). The antigen dose was 10 $\mu \mathrm{g}$, for both test formulations and controls. In the i.m. mice test groups, the test formulations and control were administered at days 0 and 28 ( 0 and 4 weeks) in the hind leg of the mice, in a volume of $0.06 \mathrm{ml}$ ( $\mathrm{HB}$ at $0.167 \mathrm{mg} / \mathrm{ml}$ ). In the i.n. mice test groups, the formulations and control were administered at days 0, 28 and 112 (0, 4 and 16 weeks), in a volume of $0.02 \mathrm{ml}$ ( $\mathrm{HB}$ at $0.5 \mathrm{mg} / \mathrm{ml}$ ) by instillation of $0.01 \mathrm{ml}$ in each nostril. Blood samples were collected in all cases from the mouse maxillary vein at days 42, 126 and 183 (corresponding to weeks 6, 18 and 26).

\subsubsection{Determination of anti-HB IgG in the serum of immunized mice by ELISA} Specific antibody levels against HB in mice sera were evaluated by ELISA at different time points. For this purpose, MaxiSorp ${ }^{\circledR}$ 96-well plates (Nunc, Denmark) were coated with a HB solution at $5 \mu \mathrm{g} / \mathrm{ml}$, in carbonate buffer (pH 9.6), and incubated overnight at $4{ }^{\circ} \mathrm{C}$. Plates were then blocked with BSA $1 \%$ in PBS for 1 hour at $37^{\circ} \mathrm{C}$.

311 In order to quantify specific anti HB-IgG, both serum samples and a mouse 
monoclonal anti-HB IgG antibody (Acris Antibodies GmbH; Hiddenhausen, Germany), were serially diluted and incubated for 2 hours at $37^{\circ} \mathrm{C}$. Afterwards, secondary antibodies (goat anti-mouse IgG from Southern Biotech, AL, USA) were added to the wells and incubated for 1 hour at $37^{\circ} \mathrm{C}$. Bound antibodies were finally revealed with 2,2'-azino-bis-3-ethylbenzothiazoline-6-sulfonic acid (ABTS) and the absorbance read at $405 \mathrm{~nm}$. To transform antibody levels in $\mu \mathrm{g} / \mathrm{ml}$ to international units (mIU/ml), a similar protocol was carried out in parallel using a control rabbit anti-HB IgG (Biokit, Barcelona, Spain) of known concentration (mIU/ml). The IgG1/IgG2a ratio was also evaluated in a pool of sera from mice immunized with HB:PARG:pIC and HB-Alum by i.m. route. In this case, the same protocol was followed but specific anti-mouse IgG1 and IgG2a secondary antibodies were used. The ratio IgG1/IgG2a was then calculated based on the absorbance levels detected. All serum samples were tested at least twice and in duplicate.

\section{Results and discussion}

Virus-like particles (VLP) antigens are having an increasing presence in the immunization protocols. Despite their good safety profile and immunogenicity, these new vaccines need to be administered in association with adjuvants in order to generate robust immune responses. Inspired by the nanoparticulate nature of the VLP, we have designed a strategy intended to increase the immune response against these antigens. This strategy relies on the use of a particulated antigen as a substrate for the adsorption of polymers with immunoadjuvant properties. The antigen chosen was the hepatitis B VLP antigen (HB), due to its nanometric size (22 nm) and negative surface charge. The biomaterials selected for the enveloping process were: protamine (PR), polyarginine (PARG), dextran sulfate (DS) and alginate (ALG). Finally, the immunomodulator poly (I:C) (pIC), was also associated to the nanostructures in order to evaluate its potential for enhancing the immune response against the enveloped antigen. With this strategy, four multi-enveloped systems (HB:PR; HB:PR:DS; HB:PARG:ALG; HB:PARG:pIC) (Figure 1) were developed, characterized and their behavior upon contact with dendritic cells and upon in vivo administration was also assessed. 


\subsection{Preparation and characterization of multi-enveloped nanoparticles}

The HB antigen in a PBS stock solution was washed with ultrapure water (by ultrafiltration) in order to decrease the salt content before its enveloping with the polymers. As the adsorption of the polymers was based on electrostatic interactions, it was important to reduce the presence of salts that could shield the electrostatic attraction between molecules of opposite charge [41]. This operation led to a decrease of the antigen sample number-distribution: before ultrafiltration it presented a size of $53 \mathrm{~nm}$ while after this process the size was of $22 \mathrm{~nm}$ (data not shown). After incubation of the antigen with the polymers, the formulation was also ultrafiltered to separate the multi-enveloped nanosystem from the free polymer and to allow the concentration of the formulation. This was considered an important step, particularly after the first coating (protamine and polyarginine), to eliminate the free polymer molecules that could, otherwise, bond with the negatively charged polymers (dextran sulfate, alginate and poly (I:C)) and interfere with the antigen enveloping process. The DLS and ELS techniques were considered the most practical techniques to characterize the antigen and to monitor its progressive coating by the polymers. In order to have a reliable signal with these techniques, the minimal HB concentration needed was $0.05 \mathrm{mg} / \mathrm{ml}$ and this concentration was taken as reference for the characterization of the multi-enveloped systems. The initial DLS intensity-distribution analysis of the antigen alone indicates a size greater than $100 \mathrm{~nm}$ (Annex 1A), which contradicts previously published work [27, 42]. However, when the DLS signal was evaluated through a volume-distribution (Annex 1B) and number-distribution analysis (Annex 1C), we observed that the majority of the HB particles in solution had in fact a size close to $22 \mathrm{~nm}(22 \pm 1)$ and that particles of this size represented the majority of the antigen volume. Such difference between intensity and number evaluation could arise from the presence of a small number of big particles resultant from HB particles agglomeration. In DLS, the intensity signal is directly proportional to the size of the particles, so even if the quantity of small particles is greater than that of large ones, the intensity-distribution profile will predominantly reflect the presence of large particles [43]. As the number-distribution size matched perfectly with the referenced size of the HB particles, this parameter was considered to be the most adequate for the antigen characterization and to follow its progressive coating through the multi-enveloping process. The presence of the referred HB particles aggregates could explain the high polydispersity (PdI 0.3). As this nanostructure was used as a template for the polymer 
adsorption, all the multi-enveloped structures developed presented also high PdI (Figure 2). The ELS analysis of the antigen alone confirmed its negative surface charge. After the addition of the protamine or polyarginine, this surface charge became positive, which suggest that the antigen surface was coated by the cationic polyamino acids. Finally, when the dextran sulfate, alginate or poly (I:C) were added to the formulations, the zeta potential detected was again negative, which indicates that the antigen particles had been coated by the anionic polymers. The adsorption of the different polymer layers led to a sequential negative-positive-negative surface charge inversion and also to a subtle increase of the size from the original $22 \mathrm{~nm}$ to about 40 $\mathrm{nm}$, upon formation of the multilayer structure.

\subsection{Determination of protamine and polyarginine adsorption and antigen enveloping efficiency}

In order to evaluate the percentage of protamine and polyarginine adsorbed onto the antigen particles, the concentration of the non-associated polymers was determined by mBCA. Under the selected experimental conditions, the percentage of polyarginine and protamine adsorbed was 23\% and 13\%, respectively (Table 1). This indicates that the amount of polymers used, exceeded the amount required for the enveloping process. These cationic polymers are hydrophilic and the mechanism driving their adsorption is based on their ionic interaction with the negatively charged surface of the antigen, as illustrated in Figure 1.

The amount of HB antigen coated by the polymers was determined by ELISA. Interestingly, although the amounts of protamine and polyarginine adsorbed to HB were quite different, the HB enveloping efficiency was relatively high (> 60\%) for both polyamino acids (Table 1). A possible explanation for the higher amount of polyarginine adsorbed could be the greater density of positive charges in this polymer. In addition, the higher molecular weight of polyarginine ( $5-15 \mathrm{kDa})$ vs. protamine (5 $\mathrm{kDa}$ ) could have also influenced the amount of polymer adsorbed. The slightly larger size and higher positive surface charge identified for HB:PARG compared with the HB:PR also support this hypothesis (Figure 2). 


\subsection{Stability of multi-enveloped particles in aqueous suspension}

A critical requisite of colloidal carriers is the preservation of their stability upon storage and also upon incubation in simulated biological fluids. Indeed, very small nanoparticles, especially those formed by ionic complexation, often have a tendency to destabilize under such conditions. Interestingly, irrespective of the nature of the coating polymer, the nano-enveloped particles remained stable in terms of particle size distribution, upon storage for at least one month at $4{ }^{\circ} \mathrm{C}$ (Annex 2). On the other hand, the behavior of the different nanostructures upon incubation in PBS at $37^{\circ} \mathrm{C}$, was slightly different. The HB:PR formulation showed a slight and rapid size increase (from 40 to $60 \mathrm{~nm}$ size) (Figure 3A) upon incubation in PBS. However, following this initial increase, the size and also the PdI remained stable for the rest of the assay. On the other hand, the HB:PR:DS prototype exhibited an adequate stability in PBS (Figure 3B) while the HB:PARG:ALG system, aggregated rapidly when dispersed in this media. Finally, the HB:PARG:pIC preserved its size and PdI for up to 4 hours in PBS (Figure 3C). The different behavior of HB:PARG:ALG and HB:PARG:pIC systems (which share the same HB:PARG core) in PBS, could be related to the different ionic forces between the first and second enveloping layers. The great negative charge density of poly (I:C) might be responsible for the strong association between these two layers.

Overall, the conclusion is that the stability of the multi-enveloped antigen nanoparticles is dependent on the balance between the attractive forces of the polymerpolymer and polymer-antigen layers, and between the polymer layer and the components of the incubation medium.

\subsection{In vitro internalization studies of fluorescently labeled multi-enveloped particles}

As indicated in the introduction, in order to facilitate the antigen presentation to the immune system, nanoparticles should have the capacity to enter the APCs. To test the conditions for this internalization process, the HB:PR:DS and HB:PARG:ALG nanosystems were prepared using TAMRA-labeled polymers and incubated with macrophages (RAW 264.7). The dye was covalently linked to the polyamino acids prior to the enveloping process. The DLS and ELS analysis of the labeled formulations confirmed that all the physicochemical parameters of the multilayer systems remained 
unaltered, with only a small decrease in the zeta potential values of the HB:PARG:ALG formulation (Annex 3).

The internalization process of the nanosytems in macrophages was visualized by confocal microscopy. The images obtained show that both are internalized, with a more pronounced uptake of the HB:PR:DS nanoparticles as compared to the HB:PARG:ALG ones (Figure 4).

\subsection{In vivo studies}

The ability of the HB:PR, HB:PR:DS and HB:PARG:ALG or HB:PARG:pIC formulations to promote a systemic humoral immune response by i.n. or i.m. routes against the hepatitis B antigen was assessed in three different in vivo trials with $\mathrm{BALB} / \mathrm{c}$ mice. The antigen dose was $10 \mu \mathrm{g}$ both for the formulations and for the alumadsorbed antigen used as positive control (same mass ratio as in licensed vaccines) [44]. The specific anti-HB IgG concentration $(\mu \mathrm{g} / \mathrm{ml})$ in mouse sera was determined by ELISA and converted to international units $(\mathrm{mIU} / \mathrm{ml})$ to permit its comparison with the protective anti-HB IgG levels observed in humans (10 mIU/ml) [45].

Following intramuscular immunization, it was found that the prototypes investigated, led to a steady anti-HB IgG profile close to $100 \mathrm{mIU} / \mathrm{ml}$ (values between 179 and 62 $\mathrm{mIU} / \mathrm{ml}$ ) during the whole experiment (Figure 5). Although these levels were lower than those observed for the alum-adjuvanted vaccine, it is important to mention that they were always superior to $10 \mathrm{mIU} / \mathrm{ml}$, a concentration known to be protective in humans. On the other hand, the effect of poly (I:C) in the formulation was found only at early times.

When testing the formulations by intranasal route and in order to better understand the adjuvant effect of enveloping the antigen, the antigen alone was also administered by the i.n. route. Despite being a highly immunogenic antigen, the HB alone generated almost undetectable IgG titers ( $2 \mathrm{mIU} / \mathrm{ml}$ ) by this route. Overall, the responses were similar for the different prototypes. The IgG concentration levels were low (below 10 $\mathrm{mIU} / \mathrm{ml}$ ) when measured at short time periods, however, they increased over time, reaching IgG levels close to $60 \mathrm{mIU} / \mathrm{ml}$ (Figure 6). These values were also significantly higher than those attained when using the free antigen. 
481 The modest humoral response observed for all the multi-enveloped systems, suggests

482 that there might be an intrinsic limitation in the multi-enveloping strategy for the hepatitis B antigen. This limitation might be related to the recent theory that underlines the importance of the exposure of a repetitive epitope array in the generation of an 485 effective immune response [3]. Such theory has been used to justify the superiority of 486 VLP antigens over soluble antigens [6, 7]. However, despite this, it is clear that the 487 rHBsAg antigen, as many others, needs to be associated with an adjuvant like Alum, 488 in order to activate the immune cells and generate an effective immune response. On 489 the other hand, the improved behavior of the multi-enveloped formulations as 490 compared to the VLP antigen alone, following intranasal administration, suggests that 491 the enveloping polymers exert a certain role. This role could be simply related to the capacity of polyarginine to facilitate the transport of proteins across the nasal mucosa [46] or it could also be related to the adjuvant effect of the biomaterials [29, 31, 33, 37, 39].

The theory on the limited exposure of the antigen epitopes in the enveloped systems would agree with the positive results previously obtained by our group for a prototype based on chitosan nanocapsules, which have VLP rHBsAg antigen exposed on their surface $[16,17]$. However, this theory would be in disagreement with the results of previous works that have shown that the coating of rHBsAg antigen [47] or its entrapment within polymers such as chitosan [14], may also lead to significant responses following either i.m. or i.n. administration. Therefore, given the different nature of the biomaterials and the different sizes of the vaccine nanostructures it is difficult to draw a conclusion at this point. Another explanation for the limited response obtained for the enveloped nanosystems could be related to the small size of the nanostructures. Even though we have postulated that small size could facilitate the

507 direct access of the antigen to the lymph nodes, the possibility that these ultra-small

508 particles would be less recognizable by the antigen-presenting cells, cannot be 509 discarded.

511 Finally, based on previous works [39, 40], the association of poly (I:C) to the 512 nanostructure was expected to lead to an enhanced response. Keeping in mind that this 513 immunostimulant would mainly have an impact on the cellular responses (associated 514 to $T_{h} 1$ responses) [48], the IgG1/IgG2a ratios of the mice immunized with the 
515 HB:PARG:pIC formulation were compared with those of the alum-absorbed antigen

516 (Figure 7). As the IgG1 and IgG2a are characteristic of a $T_{h} 2$ and $T_{h} 1$ biased-

517 response, respectively [49], we assumed that a IgG1a/IgG2 ratio superior to 1 was

518 indication of a $T_{h} 2$ polarized response while a IgG1/IgG2a ratio inferior to 1 was the

519 indication of a $\mathrm{T}_{h} 1$ polarized response [50]. The activation of lymphocytes $\mathrm{T}_{\mathrm{h}} 2$ has

520 been associated to a more preponderant humoral response, and the activation of $\mathrm{T}_{\mathrm{h}} 1$, to

521 an enhanced cellular based immunity [51]. The IgG1/IgG2a ratio found with the alum

522 absorbed antigen, indicates that the alum markedly shifted the lymphocytes towards a

$523 \mathrm{~T}_{\mathrm{h}} 2$ profile, which is a well known characteristic of this adjuvant [13]. On the

524 contrary, in the case of the HB:PARG:pIC formulation, it was found that the initially

525 balanced $\mathrm{T}_{\mathrm{h}} 1 / \mathrm{T}_{\mathrm{h}} 2$ response (IgG1a/IgG2 $\left.=0.97\right)$ shifted towards a clear polarization of

526 a $\mathrm{T}_{\mathrm{h}} 1$ response $(\mathrm{IgG} 1 \mathrm{a} / \operatorname{IgG} 2=0.54)$ at the end of the assay. The increased activation

527 of $\mathrm{T}_{\mathrm{h}} 1$ cells by the HB:PARG:pIC formulation can be an interesting advantage of this

528 system, as these cells stimulate the development of cellular immune responses capable

529 of eliminating virus infected cells [52].

530

531 4. Conclusion

532 Here we present the design and evaluation of a new strategy for the immunomodulation of immune responses associated to particulated antigens. The strategy involves using the HB particulated antigen as a model template, enveloped with a number of biomaterials and immunostimulators. The HB multi-enveloped systems were capable of generating protective IgG titers (>10 mIU/ml), but the IgG titers achieved were lower than those obtained when using alum-adsorbed antigen.

538 There were no main differences between the activity of the HB:PR, HB:PR:DS and 539 HB:PARG:ALG systems, despite their diverse composition and structure, which 540 suggests an intrinsic limitation of this multi-enveloping strategy. Nevertheless, the 541 association of the immunostimulant poly (I:C) with the enveloped antigen led to a 542 more balanced $\mathrm{T}_{\mathrm{h}} 1 / \mathrm{T}_{\mathrm{h}} 2$ response as compared to the one observed for the alum543 absorbed antigen. This could be taken as an indication of enhanced cellular response, 544 which would be of importance for the complete elimination of viral infections.

545 Overall, this work provides new insight on the properties that determine the behavior

546 of the antigen delivery nanocarriers and suggests that a very small size might not be a

547 good option for enhancing the immune response against particulated antigens. 


\section{Acknowledgments}

550 This work was supported by grants from the Bill \& Melinda Gates Foundation (Grand

551 Challenges in Global Health initiative), the Xunta de Galicia (Competitive Reference

552 Groups), the Spanish Ministry of Economy and Competitiveness (SAF2011-30337-

553 C02-02) and the FP7/REGPOT-2012-2013.1-BIOCAPS-316265. We would like to

554 thank Shantha Biotechnics Ltd. (Hyderabad, India) for providing us the HB. Jorge

555 Pinto and Mercedes Peleteiro acknowledge fellowships from the Spanish Ministry of

556 Education (FPU predoctoral grants). The technical assistance of Rafael Romero is

557 highly appreciated. 


\section{References}

1. Zepp, F., Principles of vaccine design-Lessons from nature. Vaccine, 2010. 28, Supplement 3(0): p. C14-C24.

2. Huang, D.B., J.J. Wu, and S.K. Tyring, A review of licensed viral vaccines, some of their safety concerns, and the advances in the development of investigational viral vaccines. Journal of Infection, 2004. 49(3): p. 179-209.

3. Jegerlehner, A., et al., Regulation of Ig $G$ antibody responses by epitope density and CD21-mediated costimulation. Eur J Immunol, 2002. 32(11): p. 3305-14.

4. Grgacic, E.V.L. and D.A. Anderson, Virus-like particles: Passport to immune recognition. Methods, 2006. 40(1): p. 60-65.

5. Keating, G.M. and S. Noble, Recombinant hepatitis B vaccine (Engerix-B): $a$ review of its immunogenicity and protective efficacy against hepatitis $B$. Drugs, 2003. 63(10): p. 1021-51.

6. Schiller, J.T. and D.R. Lowy, Raising Expectations For Subunit Vaccine. J Infect Dis, 2014. 24.

7. Kushnir, N., S.J. Streatfield, and V. Yusibov, Virus-like particles as a highly efficient vaccine platform: Diversity of targets and production systems and advances in clinical development. Vaccine, 2012. 31(1): p. 58-83.

8. Clements, C.J. and E. Griffiths, The global impact of vaccines containing aluminium adjuvants. Vaccine, 2002. 20, Supplement 3(0): p. S24-S33.

9. Chen, D., et al., Characterization of the freeze sensitivity of a hepatitis $B$ vaccine. Hum Vaccin, 2009. 5(1): p. 26-32.

10. Matthias, D.M., et al., Freezing temperatures in the vaccine cold chain: A systematic literature review. Vaccine, 2007. 25(20): p. 3980-3986.

11. Davaalkham, D., et al., Administration of hepatitis B vaccine in winter as a significant predictor of the poor effectiveness of vaccination in rural Mongolia: evidence from a nationwide survey. $\mathrm{J}$ Epidemiol Community Health, 2007. 61(7): p. 578-84.

12. Drucker, E., P.G. Alcabes, and P.A. Marx, The injection century: massive unsterile injections and the emergence of human pathogens. The Lancet. 358(9297): p. 1989-1992.

13. Gupta, R.K., Aluminum compounds as vaccine adjuvants. Adv Drug Deliv Rev, 1998. 32(3): p. 155-172.

14. Prego, C., et al., Chitosan-based nanoparticles for improving immunization against hepatitis B infection. Vaccine, 2010. 28(14): p. 2607-14.

15. Vila, A., et al., Low molecular weight chitosan nanoparticles as new carriers for nasal vaccine delivery in mice. Eur J Pharm Biopharm, 2004. 57(1): p. 12331. 
16. Vicente, S., et al., Co-delivery of viral proteins and a TLR7 agonist from polysaccharide nanocapsules: A needle-free vaccination strategy. Journal of Controlled Release, 2013. 172(3): p. 773-781.

17. Vicente, S., et al., A Polymer/Oil Based Nanovaccine as a Single-Dose Immunization Approach. PLoS ONE, 2013. 8(4): p. e62500.

18. Bachmann, M.F. and G.T. Jennings, Vaccine delivery: a matter of size, geometry, kinetics and molecular patterns. Nat Rev Immunol, 2010. 10(11): p. 787-796.

19. Scheerlinck, J.P. and D.L. Greenwood, Virus-sized vaccine delivery systems. Drug Discov Today, 2008. 13(19-20): p. 882-7.

20. Banchereau, J. and R.M. Steinman, Dendritic cells and the control of immunity. Nature, 1998. 392(6673): p. 245-52.

21. Pozzi, L.A., J.W. Maciaszek, and K.L. Rock, Both dendritic cells and macrophages can stimulate naive CD8 T cells in vivo to proliferate, develop effector function, and differentiate into memory cells. J Immunol, 2005. 175(4): p. 2071-81.

22. Manolova, V., et al., Nanoparticles target distinct dendritic cell populations according to their size. Eur J Immunol, 2008. 38(5): p. 1404-13.

23. Reddy, S.T., et al., In vivo targeting of dendritic cells in lymph nodes with poly(propylene sulfide) nanoparticles. Journal of Controlled Release, 2006. 112(1): p. 26-34.

24. Reddy, S.T., et al., Exploiting lymphatic transport and complement activation in nanoparticle vaccines. Nat Biotech, 2007. 25(10): p. 1159-1164.

25. Junt, T., et al., Subcapsular sinus macrophages in lymph nodes clear lymphborne viruses and present them to antiviral B cells. Nature, 2007. 450(7166): p. 110-114.

26. Correia-Pinto, J.F., N. Csaba, and M.J. Alonso, Vaccine delivery carriers: insights and future perspectives. Int J Pharm, 2013. 440(1): p. 27-38.

27. Zhao, Q., et al., Maturation of Recombinant Hepatitis B Virus Surface Antigen Particles. Human Vaccines, 2006. 2(4): p. 174-180.

28. Buschle, M., et al., Transloading of tumor antigen-derived peptides into antigen-presenting cells. Proceedings of the National Academy of Sciences, 1997. 94(7): p. 3256-3261.

29. Mattner, F., et al., Vaccination with Poly-l-Arginine As Immunostimulant for Peptide Vaccines: Induction of Potent and Long-Lasting T-Cell Responses against Cancer Antigens. Cancer Research, 2002. 62(5): p. 1477-1480.

30. Valneva Austria GmbH, Phase II Study of Immunization With a Hepatitis $C$ Virus (HCV) Antigen Peptide Vaccine, 2008: In: ClinicalTrials.gov [Internet]. Bethesda (MD): National Library of Medicine (US). 2000- [cited 2015 March 
17] Available from: http://clinicaltrials.gov/show/NCT00602784 NLM Identifier: NCT00602784.

31. Reynolds, F., R. Weissleder, and L. Josephson, Protamine as an efficient membrane-translocating peptide. Bioconjugate Chemistry, 2005. 16(5): p. 1240-1245.

32. Martínez Gómez, J.M., et al., Surface coating of PLGA microparticles with protamine enhances their immunological performance through facilitated phagocytosis. Journal of Controlled Release, 2008. 130(2): p. 161-167.

33. Yang, D. and K.S. Jones, Effect of alginate on innate immune activation of macrophages. J Biomed Mater Res A, 2009. 90(2): p. 411-8.

34. Kurachi, M., et al., Comparison of the activities of various alginates to induce TNF-alpha secretion in RAW264.7 cells. Journal of Infection and Chemotherapy, 2005. 11(4): p. 199-203.

35. Flo, T.H., et al., Involvement of toll-like receptor (TLR) 2 and TLR4 in cell activation by mannuronic acid polymers. J Biol Chem, 2002. 277(38): p. 35489-95.

36. McCarthy, R.E., L.W. Arnold, and G.F. Babcock, Dextran sulphate: an adjuvant for cell-mediated immune responses. Immunology, 1977. 32(6): p. 963-974.

37. Kerlin, R.L. and D.L. Watson, Effect of dextran sulphate on IgG subclass of antibody in efferent popliteal lymph of sheep. Immunol Cell Biol, 1987. 5: p. 411-7.

38. Lundberg, A.M., et al., Key differences in TLR3/poly I:C signaling and cytokine induction by human primary cells: a phenomenon absent from murine cell systems. Vol. 110. 2007. 3245-3252.

39. Durand, V., et al., Shaping of adaptive immune responses to soluble proteins by TLR agonists: a role for IFN-alpha/beta. Immunol Cell Biol, 2004. 82(6): p. 596-602.

40. Sloat, B. and Z. Cui, Nasal Immunization with Anthrax Protective Antigen Protein Adjuvanted with Polyriboinosinic-Polyribocytidylic Acid Induced Strong Mucosal and Systemic Immunities. Pharmaceutical Research, 2006. 23(6): p. 1217-1226.

41. Van de Steeg, H.G.M., et al., Polyelectrolyte adsorption: a subtle balance of forces. Langmuir, 1992. 8(10): p. 2538-2546.

42. Diminsky, D., et al., Comparison between hepatitis B surface antigen (HBsAg) particles derived from mammalian cells (CHO) and yeast cells (Hansenula polymorpha): composition, structure and immunogenicity. Vaccine, 1997. 15(6-7): p. 637-47.

43. Instruments, M., Dynamic light scattering: an introduction in 30 minutes. Technical Note Malvern, MRK656-01, 2012: p. 1-8. 
44. Rustgi, V.K., C.J. Schleupner, and D.S. Krause, Comparative study of the immunogenicity and safety of Engerix-B administered at 0, 1, 2 and 12 months and Recombivax HB administered at 0, 1, and 6 months in healthy adults. Vaccine, 1995. 13(17): p. 1665-1668.

45. West, D.J. and G.B. Calandra, Vaccine induced immunologic memory for hepatitis B surface antigen: implications for policy on booster vaccination. Vaccine, 1996. 14(11): p. 1019-1027.

46. Miyamoto, M., et al., Effect of poly-l-arginine on the nasal absorption of FITC-dextran of different molecular weights and recombinant human granulocyte colony-stimulating factor (rhG-CSF) in rats. International Journal of Pharmaceutics, 2001. 226(1-2): p. 127-138.

47. Tafaghodi, M., et al., Hepatitis B surface antigen nanoparticles coated with chitosan and trimethyl chitosan: impact of formulation on physicochemical and immunological characteristics. Vaccine, 2012. 30(36): p. 5341-5348.

48. Hafner, A.M., B. Corthesy, and H.P. Merkle, Particulate formulations for the delivery of poly(I:C) as vaccine adjuvant. Adv Drug Deliv Rev, 2013. 65(10): p. 1386-99.

49. Finkelman, F.D., et al., Lymphokine control of in vivo immunoglobulin isotype selection. Annual review of immunology, 1990. 8(1): p. 303-333.

50. Cribbs, D.H., et al., Adjuvant-dependent modulation of Th1 and Th2 responses to immunization with $\beta$-amyloid. International Immunology, 2003. 15(4): p. 505-514.

51. Paul, W.E. and R.A. Seder, Lymphocyte responses and cytokines. Cell, 1994. 76(2): p. 241-251.

52. Graham, M.B., V.L. Braciale, and T.J. Braciale, Influenza virus-specific CD4+ Thelper type 2 T lymphocytes do not promote recovery from experimental virus infection. J Exp Med, 1994. 180(4): p. 1273-82. 
Figure captions:

Figure 1: Illustration of the adsorption process of the (A) protamine (PR) and (B) polyarginine (PARG) layers onto the $\mathrm{HB}$ antigen nanoparticles, and the subsequent adsorption of the dextran sulfate (DS) (HB:PR:DS), alginate (ALG) (HB:PARG:ALG) or poly (I:C) (HB:PARG:pIC) layers, respectively.

Figure 2: Evolution on the particle size (Size), polydispersity index (PdI) and surface charge (zeta potential) of the HB nanoparticles coated with multiple layers, namely (A) HB:PR and HB:PR:DS coated with protamine (PR), or PR plus dextran sulfate, respectively (B) HB:PARG and HB:PARG:ALG, coated with polyarginine (PARG) or PARG plus ALG, respectively and, (C) HB: PARG and HB:PARG:pIC, coated with polyarginine (PARG) or PARG plus poly (I:C) (pIC), respectively. Results are presented as the mean \pm standard deviation $(n=4)$.

Figure 3: Evaluation of size number distribution (Size) and polydispersity index (PdI) after 4 hours at $37^{\circ} \mathrm{C}$ in PBS of (A) HB nanoparticles coated with protamine (HB:PR), (B) HB nanoparticles coated with protamine and dextran sulfate (HB:PR:DS) and (C) HB nanoparticles coated with polyarginine and poly (I:C) (HB:PARG:pIC). Results are presented as the mean \pm standard deviation $(\mathrm{n}=3)$.

Figure 4: Confocal microscopy analysis of the uptake of fluorescent nanoparticles: (A) HB:PR:DS and (B) HB:PARG:ALG, by RAW 264.7 macrophage cells. Red channel: TAMRA-labeled particles). Green channel: Alexa Fluor 488-phalloidin (cytoskeleton). Blue channel: DAPI (nucleus).

Figure 5: Systemic humoral (IgG) immune response observed in mice upon intramuscular ( 0 and 4 weeks) immunization with HB antigen nanoparticles coated with protamine (HB:PR), protamine and dextran sulfate (HB:PR:DS), polyarginine and alginate (HB:PARG:ALG) or polyarginine and poly (I:C) (HB:PARG:pIC). Hepatitis B antigen adsorbed to Alum (HB:Alum) was used as control. The plot represents the antiHB IgG average quantified at different time points after primary immunization. Results are shown as mean \pm standard deviation $(\mathrm{n}=10)$. 
Figure 6: Systemic humoral (IgG) immune response observed in mice upon intranasal (0, 4 and 16 weeks) immunization with $\mathrm{HB}$ antigen nanoparticles coated with protamine (HB:PR), protamine and dextran sulfate (HB:PR:DS), polyarginine and alginate (HB:PARG:ALG) or polyarginine and poly (I:C) (HB:PARG:PIC). Hepatitis B antigen dispersed in PBS (HB in PBS) was used as control. The plot represents the anti-HB IgG average quantified at different time points after primary immunization. Results are shown as the mean \pm standard deviation $(\mathrm{n}=10)$.

Figure 7: IgG1 and IgG2a subtype ratios in the sera of mice immunized with HB antigen nanoparticles coated with polyarginine and poly (I:C) (HB:PARG:pIC) (grey squares), and with HB adsorbed onto alum (HB:Alum) (black circle). Results are shown as the mean $(n=10)$.

Annex 1. Comparison of DLS signal analysis of the HB antigen nanoparticles alone through three different size distributions: (A) Intensity, (B) Volume and (C) Size

Annex 2. Evolution of size number distribution (Size) and polydispersity index (PdI) after one month storage at $4{ }^{\circ} \mathrm{C}$ of the $\mathrm{HB}$ nanoparticles coated with protamine (HB:PR), with protamine and dextran sulfate (HB:PR:DS), with polyarginine and alginate (HB:PARG:ALG) or with polyarginine and poly (I:C) (HB:PARG:pIC). Results are presented as the mean \pm standard deviation $(\mathrm{n}=3)$.

Annex 3.Comparison of the size number distribution (Size), polydispersity index (PdI) and surface charge (Zeta) of non-labeled and TAMRA-labeled HB:PR nanoparticles (A), and of non-labeled and TAMRA-labeled HB:PARG and HB:PARG:ALG formulations (B). Results are presented as mean \pm standard deviation $(n=3)$. 


\section{Figure 1}

A
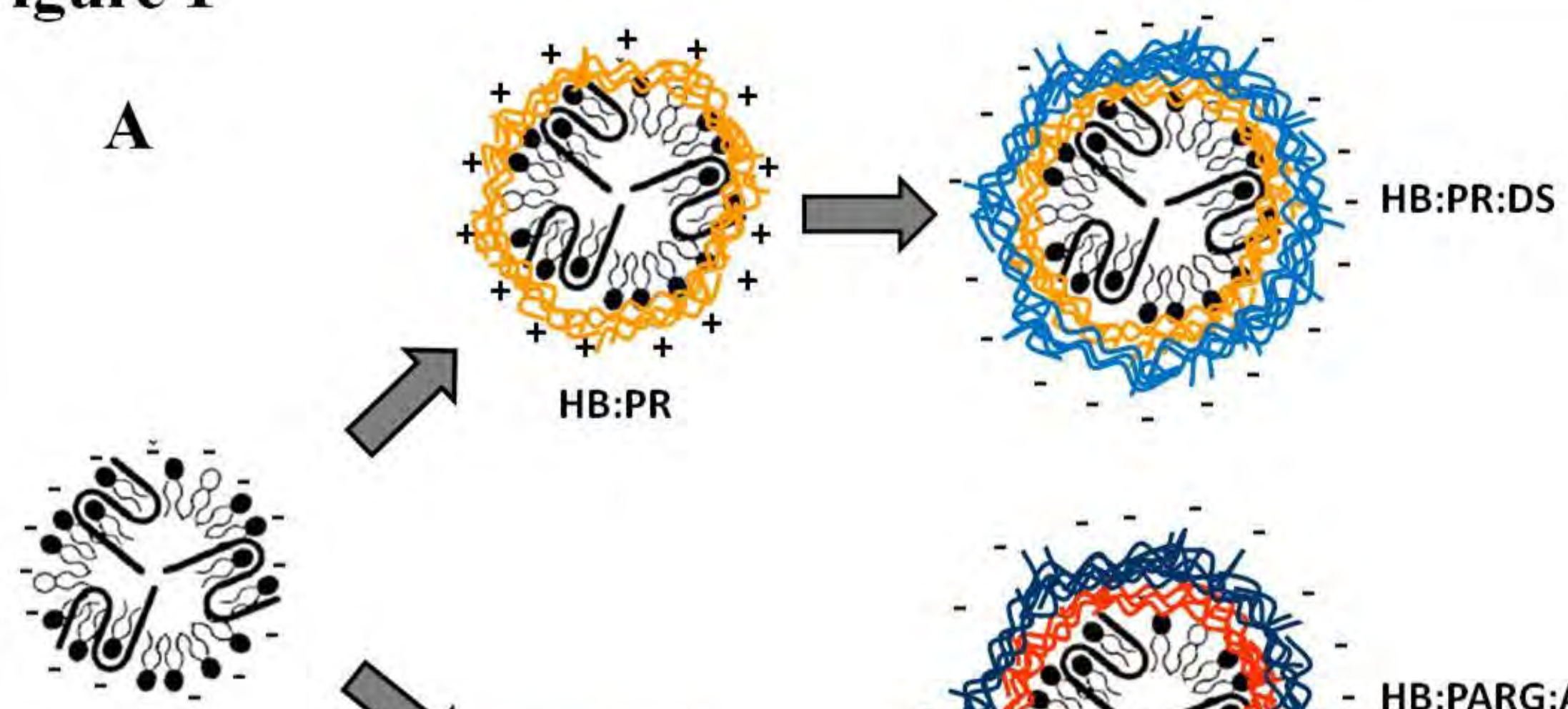

Virus-like particle

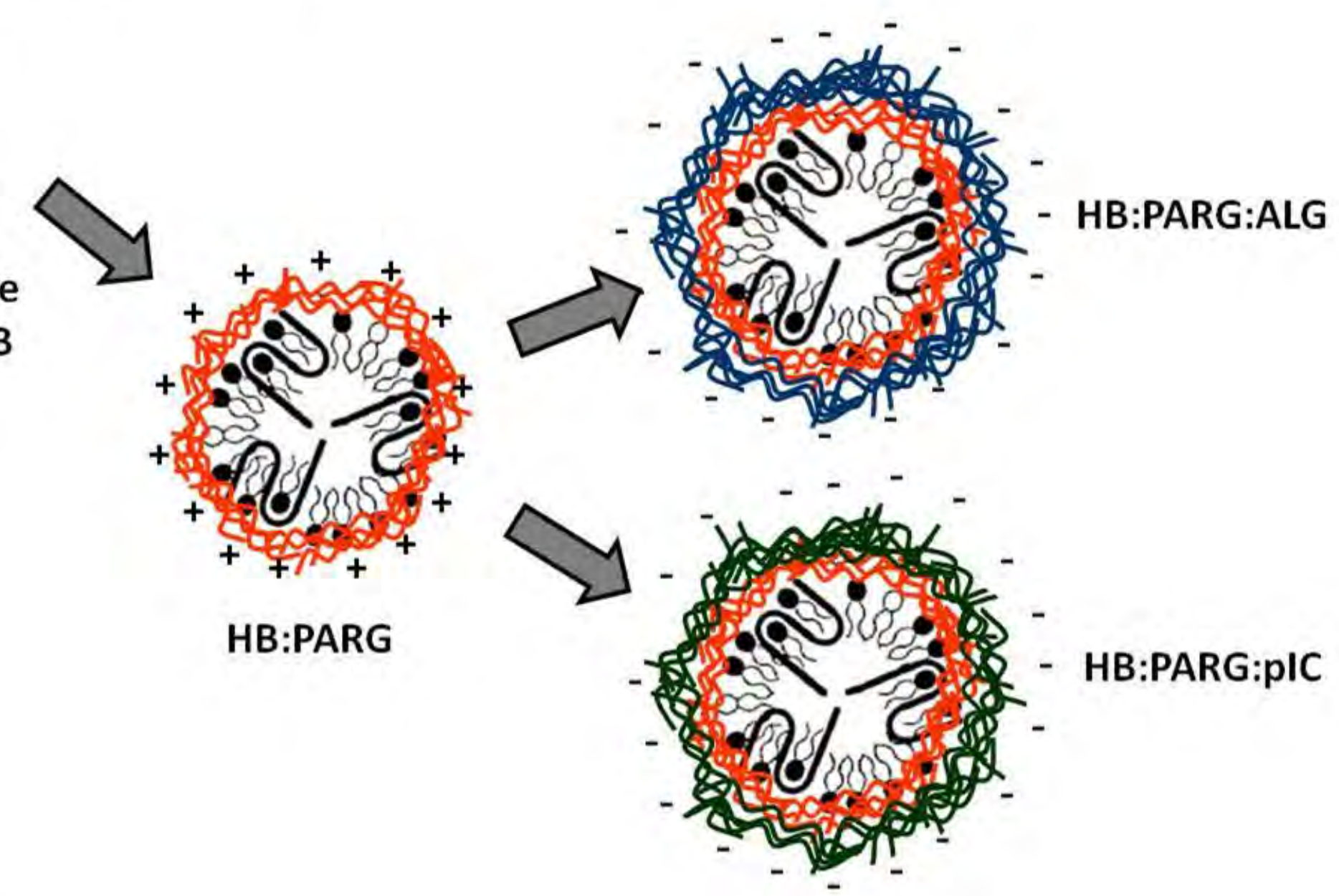

HB:PARG

(VLP) Hepatitis B

B

Polyarginine (PARG) Protamine (PR)*<smiles>CC(C)N[C@@H](CCCNC(=N)N)C(=O)C(C)(C)C</smiles>

Alginate (ALG)



Dextran Sulfate (DS)

Poly (I:C) (pIC)



\section{Figure 2}

A

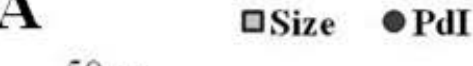

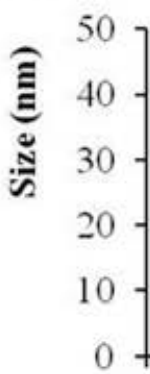

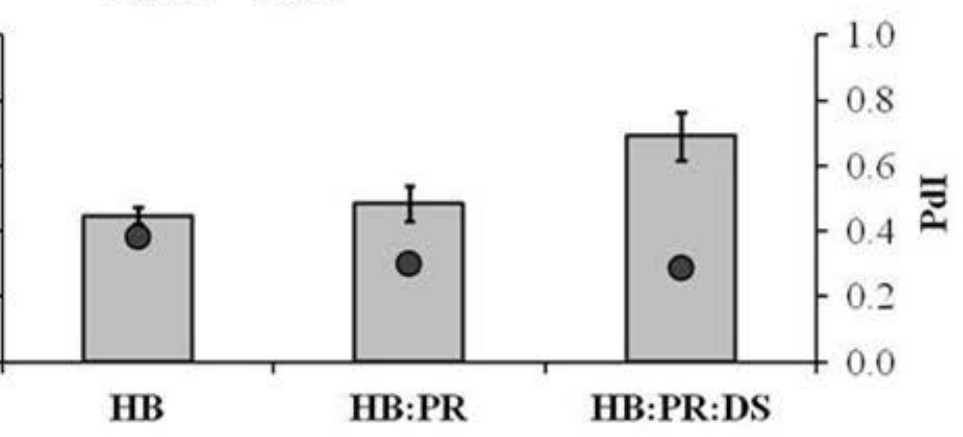

B

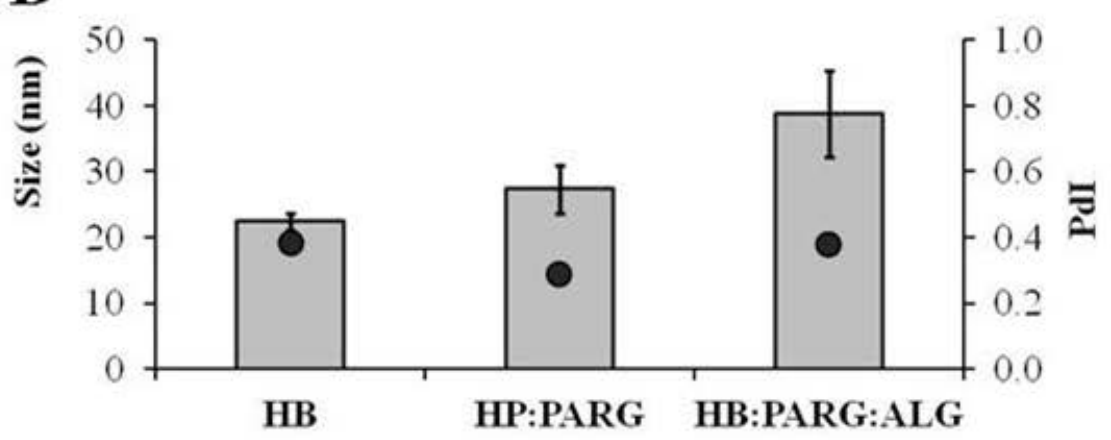

C



$-\bullet$ Zeta potential


$\left.\begin{array}{l}1 \\ 0 \\ - \\ -\end{array}\right]$



$\mathrm{HB}$

HB:PR:DS

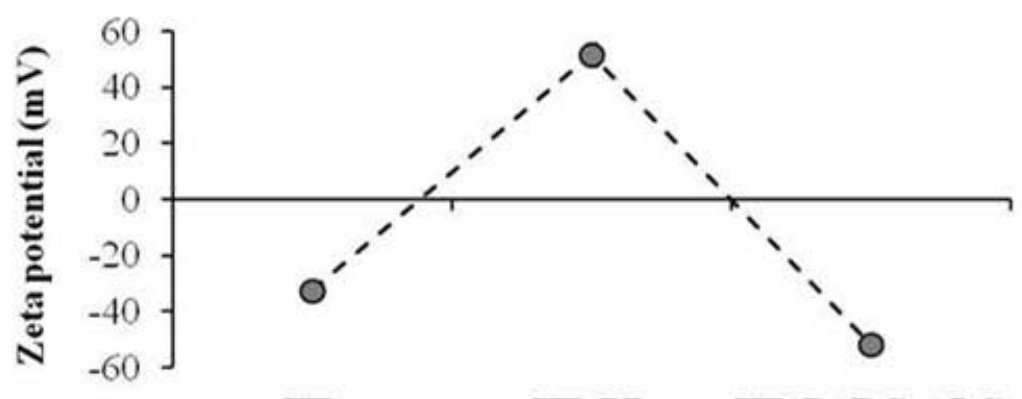

$\mathrm{HB}$
HB:PR HB:PARG:ALG

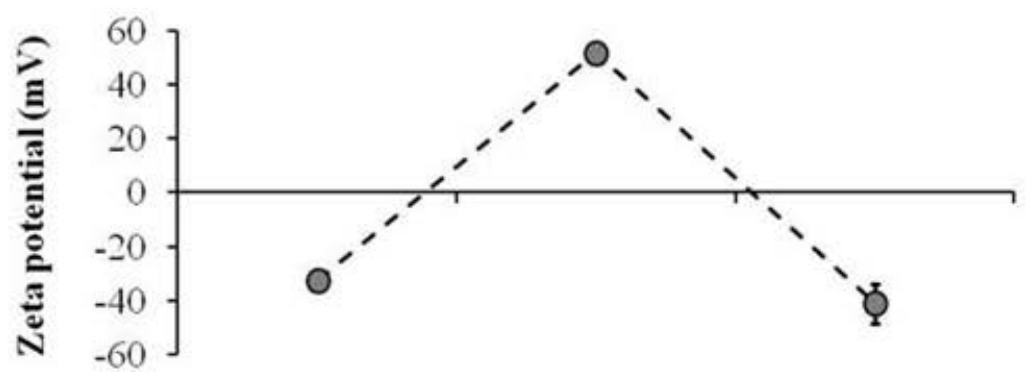

HB

HP:PARG HB:PARG:pIC 


\section{Figure 3}

A
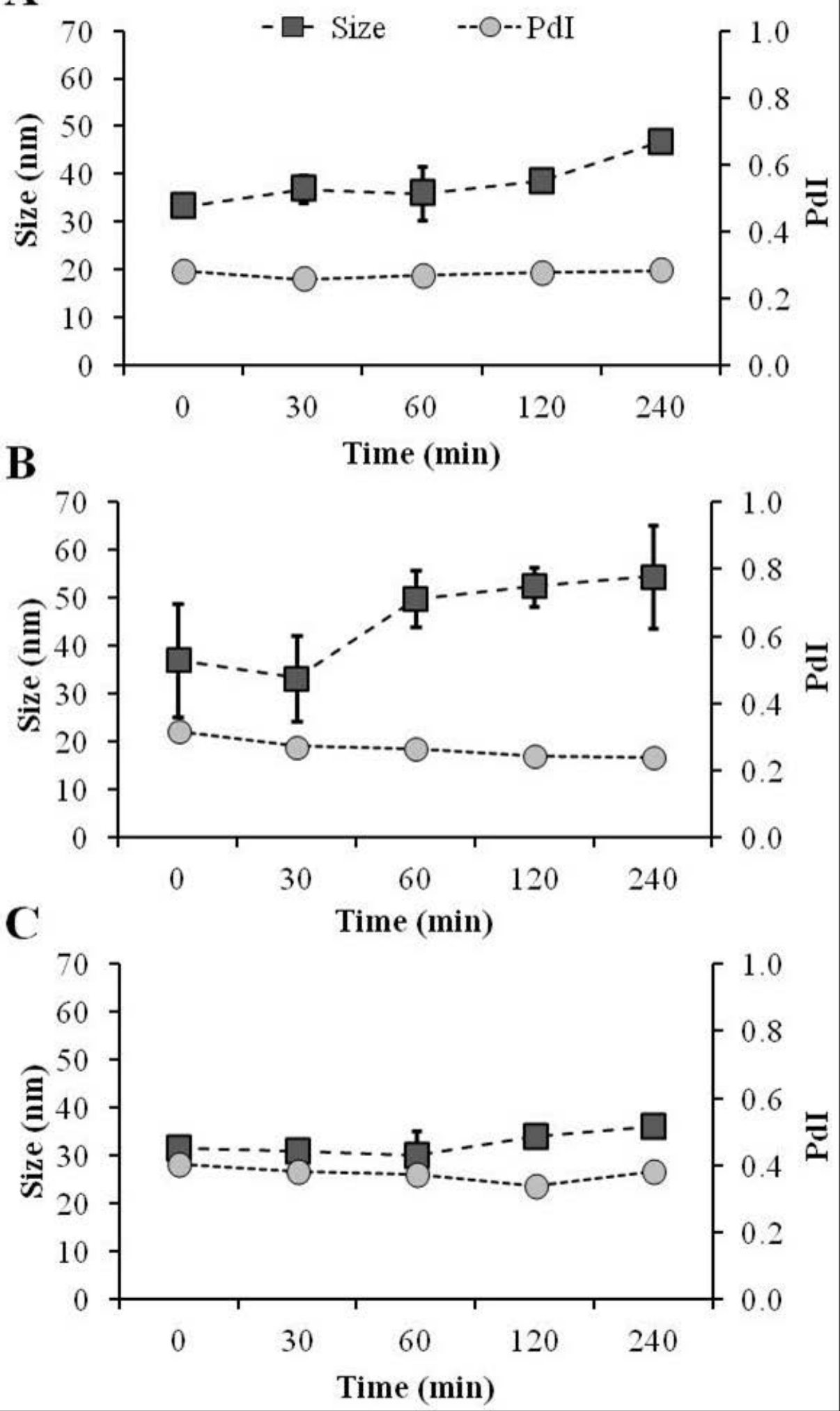


\section{Figure 4}



\section{Figure 5}

口HB:PR Nanoparticles, I.M.

口HB:PR:DS Nanoparticles, I.M.

口HB:PARG:ALG Nanoparticles, I.M.

口HB:PARG:pIC Nanoparticles, I.M.

四

口HB:Alum, I.M.

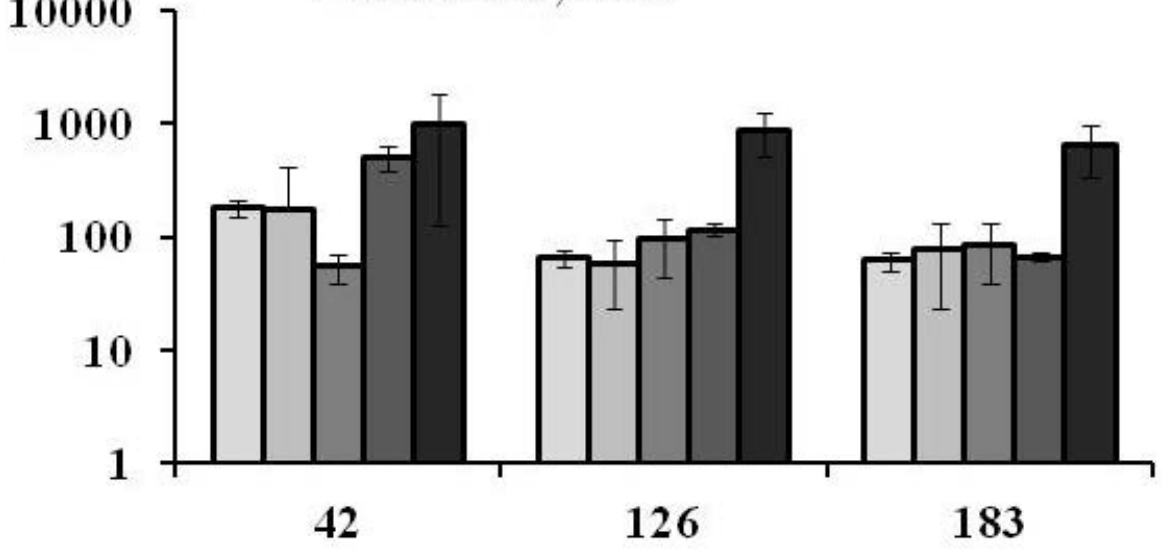

Time (days) 


\section{Figure 6}

口HB:PR Nanoparticle, I.N.

口HB:PR:DS Nanoparticle, I.N.

口HB:PARG:ALG Nanoparticle, I.N.

口HB:PARG:pIC Nanoparticle, I.N.

口HB in PBS, I.N.

(3)
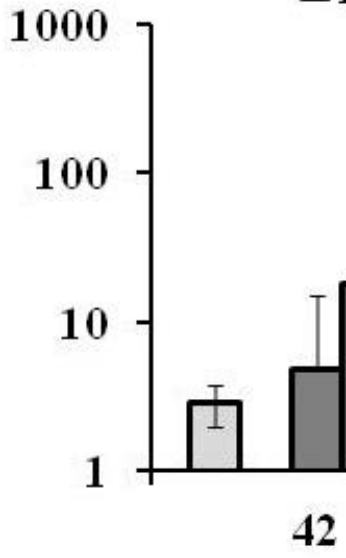

42

126

183

Time (days) 
Figure 7

$$
\text { - }- \text { - HB:PARG:pIC }
$$

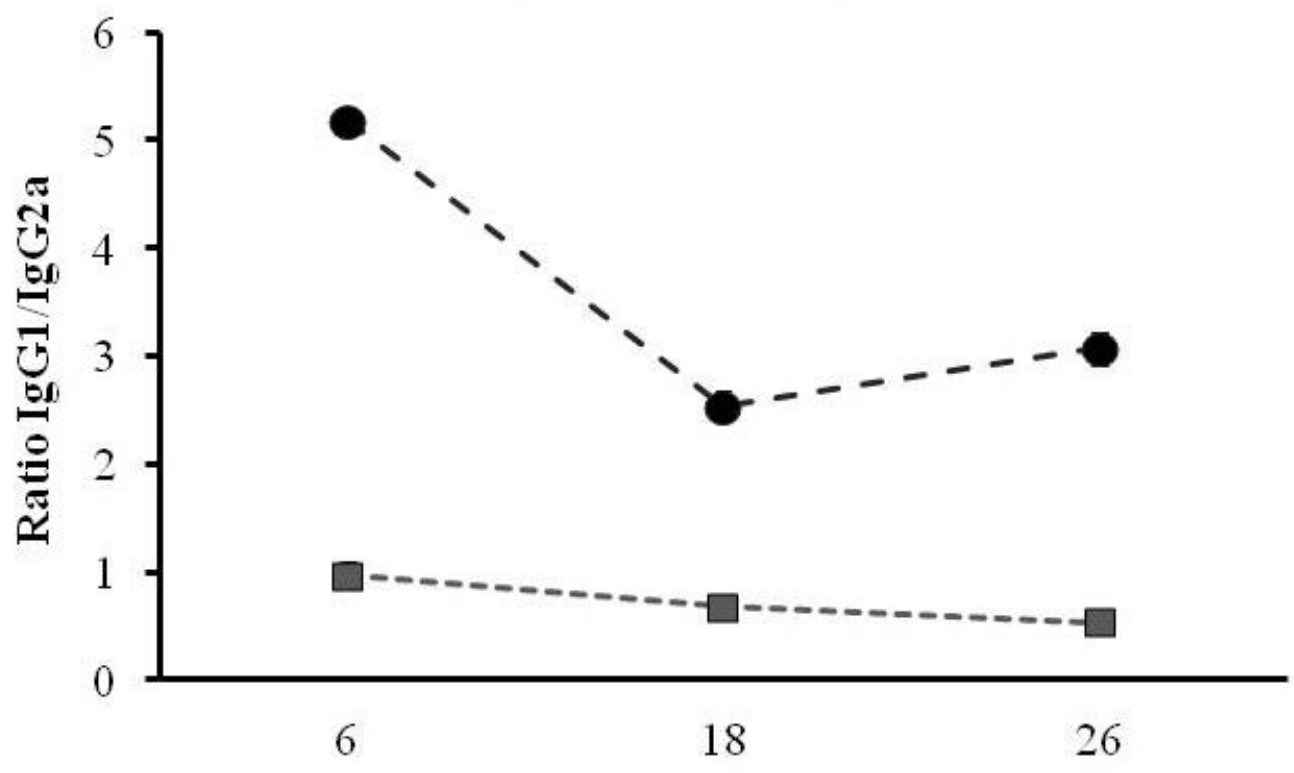

Time (weeks) 


\section{Annex 2}

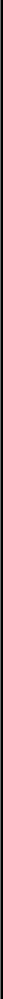




\section{Annex 3}
A
口Size
口Size Labeled
B
口Size
口Size Labeled
O PdI
- PdI Labeled
O PdI
- PdI Labeled
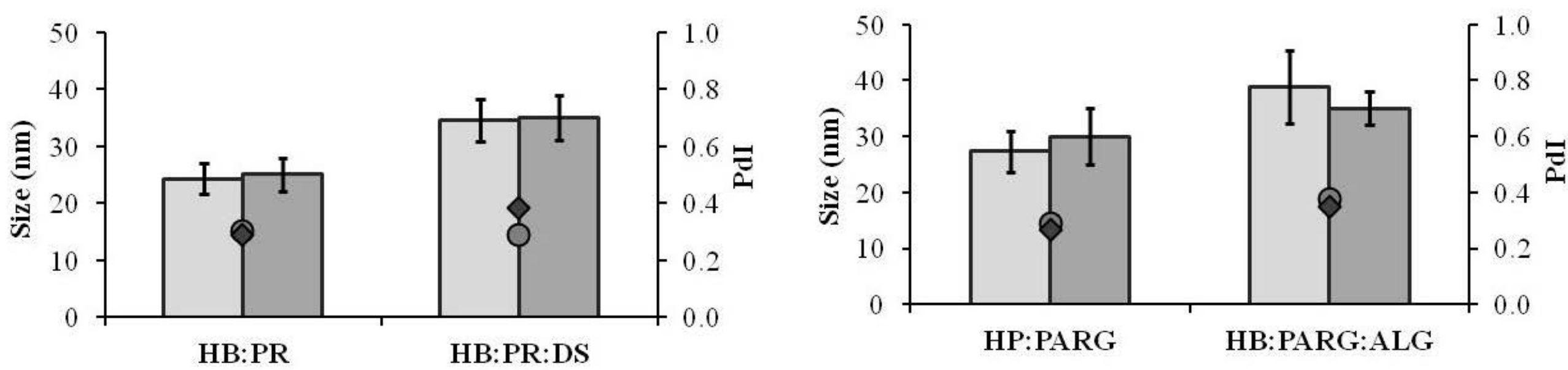



口Zeta

口Zeta Labeled

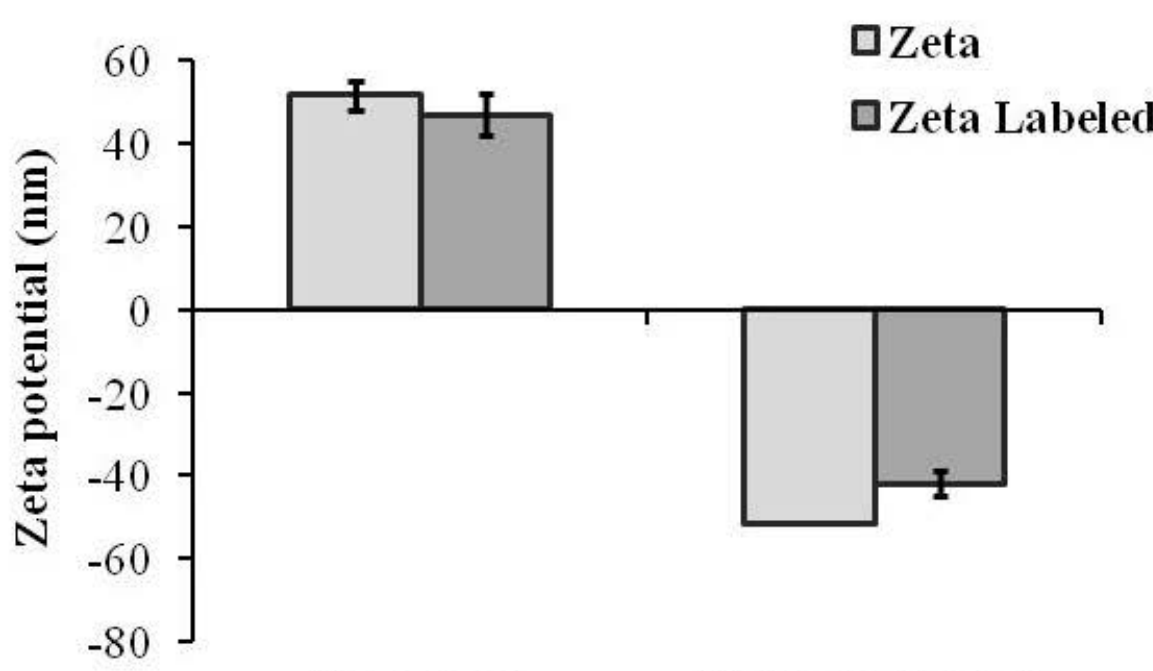

HP:PARG

HB:PARG:ALG 


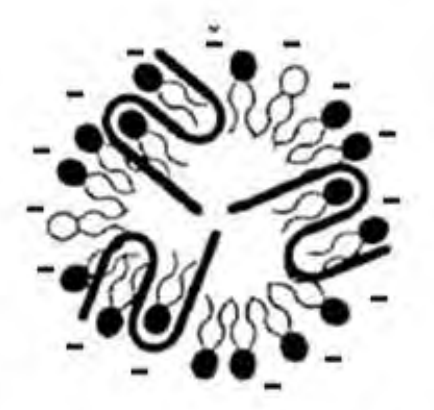

Virus-like particle Hepatitis B (HB)

PR: Protamine

PARG: Polyarginine

DS: Dextran sulfate

ALG: Alginate

pIC: $\quad$ Poly (I:C)

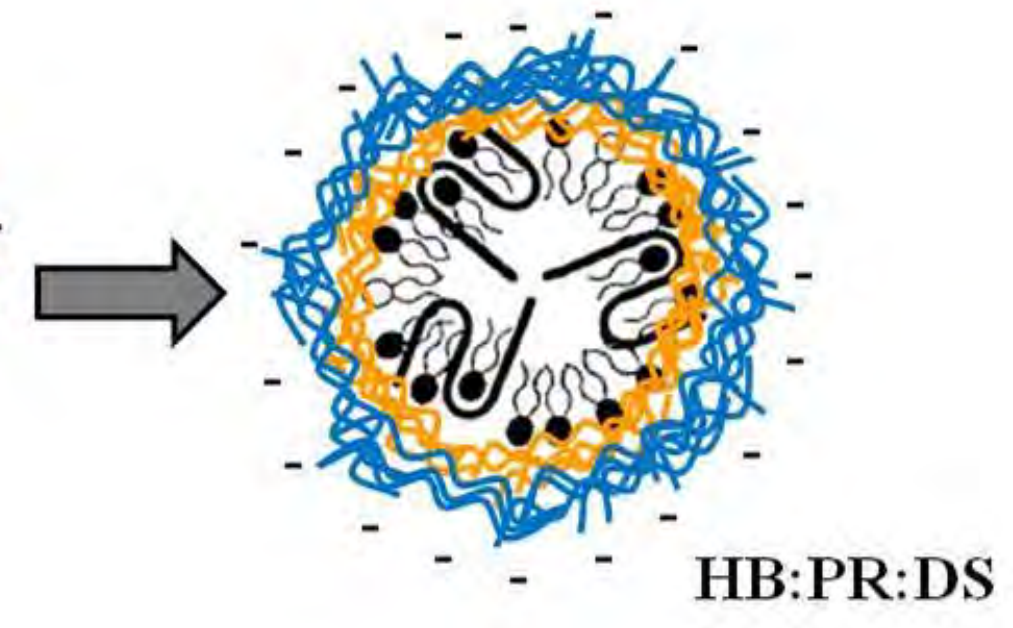

HB:PR

HB:PR:DS

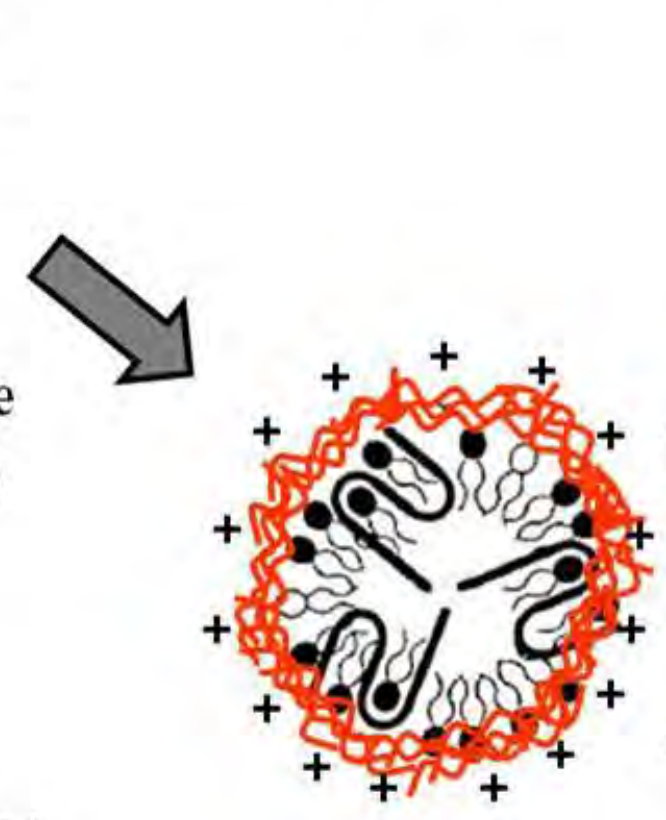

HB:PARG

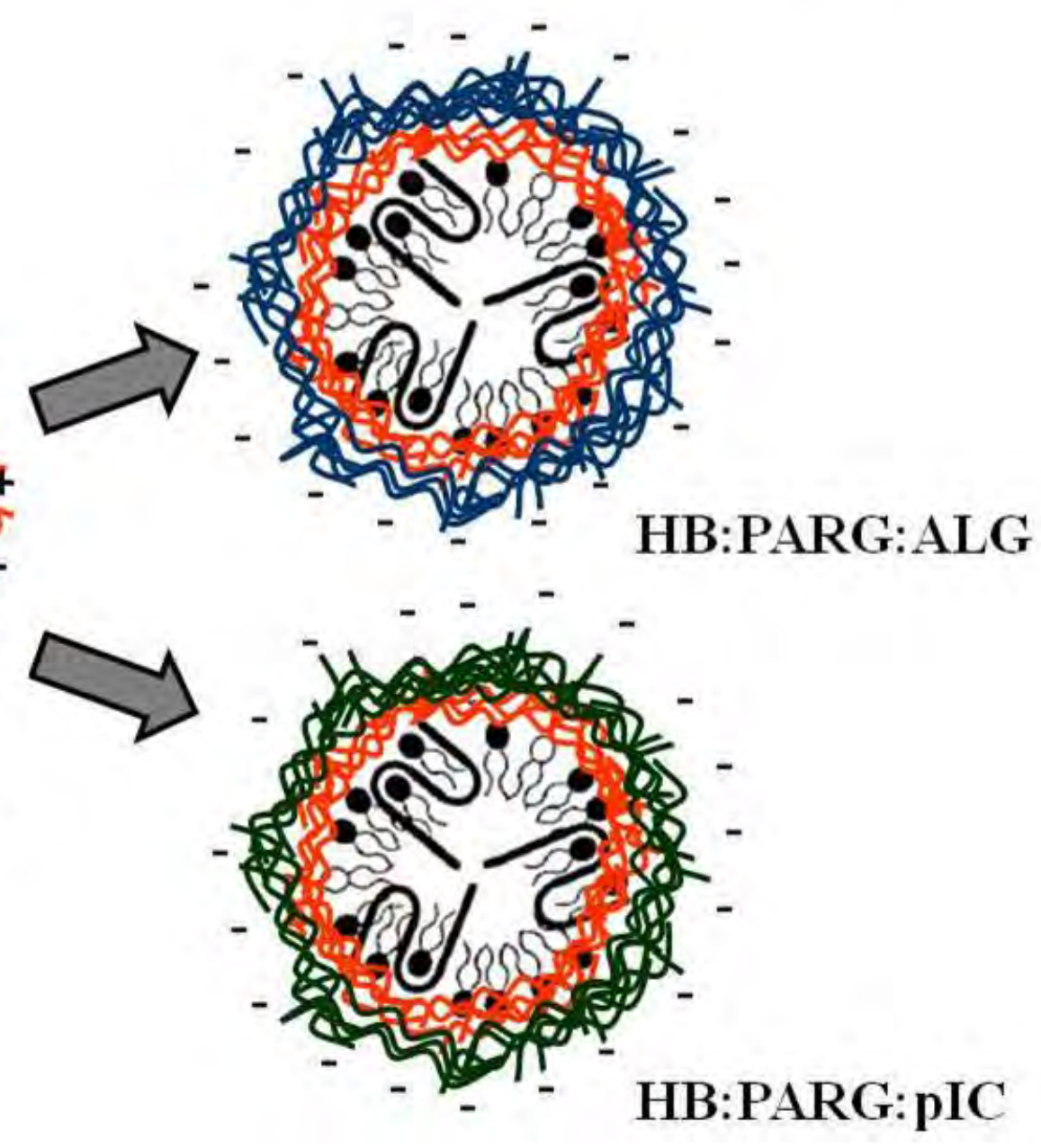

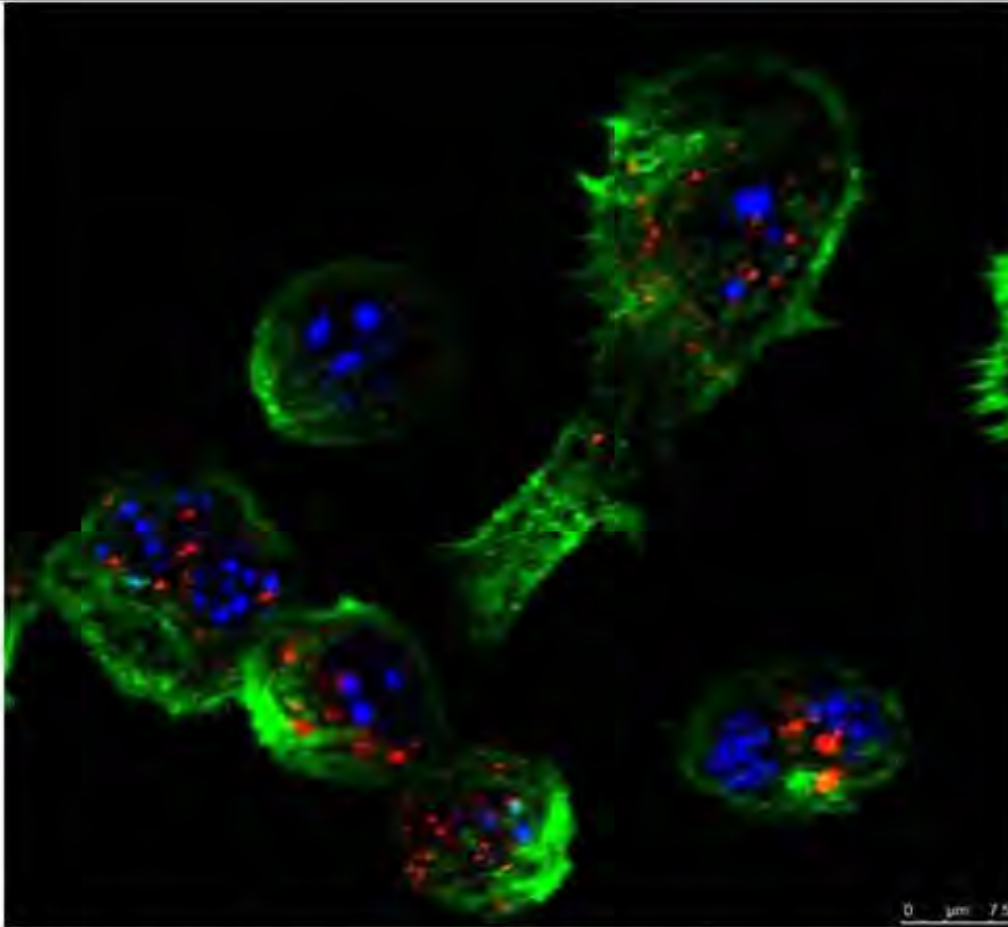

Internalization of fluorescent multienveloping nanoparticles (Red)

Increased IgG response Balanced $T_{h 1} 1 / T_{12} 2$ response

-글-HB:PARG:pIC - HB:Alum

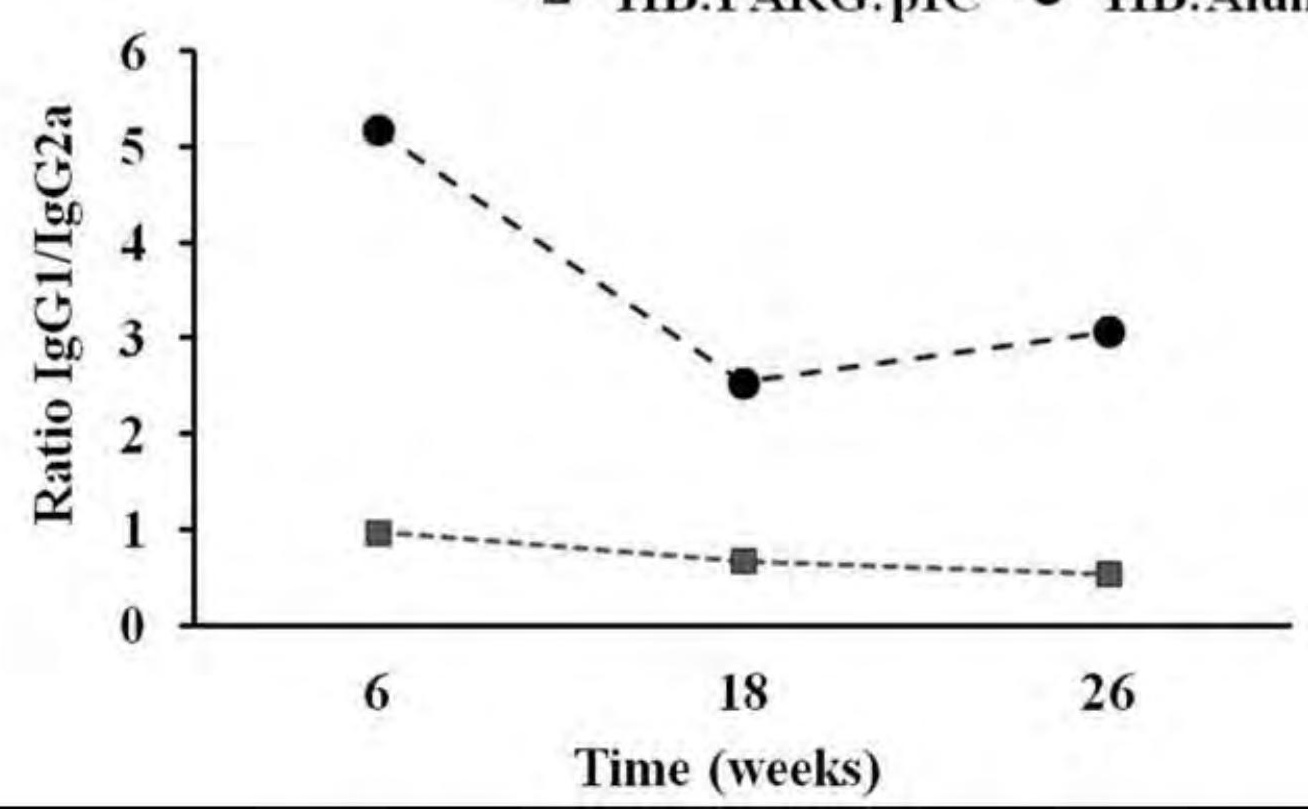

